\title{
ETHzürich
}

ETH Library

\section{Mechanical loading of mouse caudal vertebrae increases trabecular and cortical bone mass-dependence on dose and genotype}

\author{
Journal Article \\ Author(s): \\ Webster, Duncan J.; Wasserman, Elad; Ehrbar, Martin; Weber, Franz; Bab, Itai; Müller, Ralph (D) \\ Publication date: \\ 2010 \\ Permanent link: \\ https://doi.org/10.3929/ethz-b-000024162 \\ Rights / license: \\ In Copyright - Non-Commercial Use Permitted \\ Originally published in: \\ Biomechanics and Modeling in Mechanobiology 9(6), https://doi.org/10.1007/s10237-010-0210-1
}




\title{
Mechanical loading of mouse caudal vertebrae increases trabecular and cortical bone mass-dependence on dose and genotype
}

\author{
Duncan Webster • Elad Wasserman • Martin Ehrbar • \\ Franz Weber • Itai Bab • Ralph Müller
}

Received: 17 December 2009 / Accepted: 9 March 2010 / Published online: 30 March 2010

(C) Springer-Verlag 2010

\begin{abstract}
Most in vivo studies addressing the skeletal responses of mice to mechanical loading have targeted cortical bone. To investigate trabecular bone responses also we have developed a caudal vertebral axial compression device (CVAD) that transmits mechanical loads to compress the fifth caudal vertebra via stainless steel pins inserted into the forth and sixth caudal vertebral bodies. Here, we used the CVAD in C57BL/6 (B6) and C3H/Hej (C3H) female mice (15 weeks of age) to investigate whether the effect of regular bouts of mechanical stimulation on bone modeling and bone mass was dependent on dose and genotype. A combined micro-computed tomographic and dynamic histomorphometric analysis was carried out at the end of a 4-week loading regimen $(3,000$ cycles, $10 \mathrm{~Hz}, 3 \mathrm{x}$ week) for load amplitudes of $0 \mathrm{~N}, 2 \mathrm{~N}, 4 \mathrm{~N}$ and 8 N. Significant increases in trabecular bone mass of 9 and $21 \%$ for loads of $4 \mathrm{~N}$ and $8 \mathrm{~N}$, respectively, were observed in B6 mice. A significant increase of $10 \%$ in trabecular bone mass occurred for a load of $8 \mathrm{~N}$ in the $\mathrm{C} 3 \mathrm{H}$ strain. For other loads, no significant increases were detected. Both mouse strains exhibited substantial increases in trabecular bone formation rates for all loads, B6: $111 \%(2 \mathrm{~N}), 86 \%(4 \mathrm{~N}), 164 \%$ (8N), C3H: $41 \%(2 \mathrm{~N}), 38 \%(4 \mathrm{~N}), 141 \%(8 \mathrm{~N})$. Significant decreases in osteoclast number of 146 and $93 \%$ for a load of
\end{abstract}

D. Webster · E. Wasserman $\cdot$ R. Müller $(\bowtie)$

Institute for Biomechanics, ETH Zürich,

Wolfgang-Pauli-Strasse 10, 8093 Zürich, Switzerland

e-mail: ram@ethz.ch

URL: http://www.biomech.ethz.ch

E. Wasserman · I. Bab

Bone Laboratory, The Hebrew University of Jerusalem,

Jerusalem, Israel

M. Ehrbar · F. Weber

Department of Craniomaxillofacial Surgery,

University of Zürich, Zürich, Switzerland
$8 \mathrm{~N}$ were detected in $\mathrm{B} 6$ and $\mathrm{C} 3 \mathrm{H}$ mice, respectively. These findings demonstrate that the effect of loading on the structural and functional parameters of bone is dose and genotype dependent. The caudal vertebral loading model established here is proposed for further studies addressing the molecular processes involved in the skeletal responses to mechanical stimuli.

Keywords Mechanical loading - Trabecular bone . Cortical bone $\cdot$ Bone adaptation · Bone morphometry

\section{Introduction}

Mechanical loading is perhaps the most important environmental determinant of bone mass and functional integrity. It is well established that insufficient mechanical stimuli, as in the case of prolonged bed rest and hypogravity, lead to massive bone loss. Conversely, it has also been demonstrated that mechanical overloading results in enhanced bone formation and a net gain in bone mass (Rubin and Lanyon 1985; Duncan and Turner 1995; Biewener et al. 1996; Evans 1998). These stimuli affect both cortical bone and trabecular bone. Trabecular bone, in particular, has been shown to have a more enduring sensitivity to mechanical stimulation in human adults (Albright 1987; Frost 1987). Osteoporosis, the most prevalent degenerative disease in western societies, has been partly attributed to a reduction in muscle mass and function and consequently decreased mechanical usage of the skeleton (Marcus 1995). Hence, it is anticipated that an understanding of the processes involved in the skeletal response to mechanical forces could lead to the identification of molecular targets for the development of anti-osteoporotic therapies. 
The availability of in vivo models for load-regulated bone adaptation is key to understanding the underlying biochemical mechanisms. To study cortical bone, several studies have established in vivo mouse models, which demonstrate significant increases in bone formation in response to dynamic load regimens applied to mice tibia and ulna (Akhter et al. 1998; Robling and Turner 2002; Kuruvilla et al. 2008). These models have been used to investigate the biochemical pathways associated with load-induced cortical bone adaptation using C57BL/6 and C3H/Hej inbred strains (Kesavan et al. 2005; Xing et al. 2005; Lau et al. 2006). These strains are of particular interest as they exhibit a number of contrasting phenotypes specific to both cortical and trabecular bone, including the mechano-sensitivity of cortical bone (Kodama et al. 2000; Turner et al. 2000; Robling and Turner 2002; Koller et al. 2003). Furthermore, breeding strategies have been employed together with quantitative trait loci (QTL) analysis in an effort to refine the search for the chromosomal regions responsible for the complementary phenotypes (Beamer et al. 2001; Kesavan et al. 2006). Compared to cortical bone, trabecular bone has been studied less extensively. One of the few models currently available to assess load-induced changes in trabecular bone uses a mechanical device to apply an axial compressive force to the 8th caudal vertebrae of a rat via K-wires inserted into the two, adjacent caudal vertebrae (Chambers et al. 1993; Guo et al. 2002). However, studies employing this model have been hampered by the current inaccessibility to genetic manipulations, which are available mainly in mice. A mouse model, which enabled the study of load-regulated bone adaptation in mice, would therefore be advantageous. Two studies have reported load-induced trabecular bone adaptation in mice. De Souza et al. (2005) mechanically stimulated mice tibia using dynamic loads with magnitudes between 5 and $13 \mathrm{~N}$ and demonstrated a significant increase in trabecular bone volume density when pooling all the loaded groups together. Using a similar approach Fritton et al. (2005) showed trabecular bone volume density and trabecular thickness to increase significantly by 15 and $12 \%$ when applying a single 6 week long loading regimen.

Here, we present an alternative in vivo model for the study of both cortical and trabecular bone adaptation in mice. Using an approach similar to the rat vertebra model, we hypothesize that the response of murine cortical and trabecular bone to mechanical loading is both dose and genotype dependent. Although invasive, compared to the tibia loading model the proposed model has the advantage that much more trabecular bone can be analysed. Preliminary calculations show that the C5 caudal vertebrae from female B6 mice aged 19 weeks contains 50\% more trabecular bone mass than tibiae from the same mice. This will prove useful in future studies, which investigate load-regulated genes as the sensitivity of current gene detection technologies is limited by the amount of input material. Furthermore, owing to the size and shape of the
C5 caudal vertebrae the proposed loading configuration will result in a much simplified micromechanical environment i.e. the presence of bending stresses and shear stresses will be minimized, which is not the case with long bones. This simplified modality of compression will enable us to more easily characterize the spatial relationship between loadinduced bone adaptation the associated micromechanical environment.

The objectives of this study are twofold: (1) To demonstrate that the amount of load-induced bone mass increases with increasing mechanical load in both cortical and trabecular compartments and (2) To compare the skeletal responsiveness to mechanical stimulation in both $\mathrm{B} 6$ and $\mathrm{C} 3 \mathrm{H}$ mouse strains.

\section{Materials and methods}

\subsection{Mechanical loading apparatus}

To mechanically load murine vertebrae, a dual-axis closedloop feedback device was used. This device has been developed to apply a precisely controlled cyclical, compressive load to the fifth caudal vertebrae (C5) of mice at a frequency of $10 \mathrm{~Hz}$ via stainless steel pins surgically inserted into the fourth (C4) and sixth caudal (C6) vertebrae (Fig. 1). Surgical insertion of the stainless steel pins was performed using a special pinning device, compatible with X-ray fluoroscopy. The device makes use of a V-clamp to simultaneously secure and automatically locate the cranio-caudal axis of the mouse tail. The pins are loaded into channels integral to the V-clamp and are manually pushed through the centres of the vertebrae, perpendicular to the cranial-caudal axis. A digital mobile C-arm (OEC MiniView 6800, GE Medical Systems, Glattbrugg, Switzerland) was used to locate C4 and C6 for pinning. In a previous study (Webster et al. 2008) when attaching, in vitro, micro-strain gages (EA-06-015DJ120, Vishay Micro-Measurements, Malvern, PA) to ventral and dorsal cortical surfaces of $\mathrm{C} 5$ vertebrae, the loading configuration described here was shown to induce reproducible axial strains: When mechanically loading four different B6 C5 caudal vertebrae ( 15 weeks of age), in vitro, at $2 \mathrm{~N}, 4 \mathrm{~N}$ and $8 \mathrm{~N}$ for 3,000 cycles at a frequency of $10 \mathrm{~Hz}$ the mean peak axial strains were found to be $239 \pm 50 \mu \varepsilon, 434 \pm 77 \mu \varepsilon$ and $798 \pm 114 \mu \varepsilon$ respectively.

\subsection{Experimental design}

Female mice, $40 \mathrm{~B} 6$ and $40 \mathrm{C} 3 \mathrm{H}, 9$ weeks of age (Harlan Ltd.) were housed in a husbandry unit in groups of 5 and given 3 weeks to acclimatize to their new environment. At the end of the period of acclimatization, all mice had stainless steel insect pins $(0.5 \mathrm{~mm}$ diameter, Fine Science Tools, 


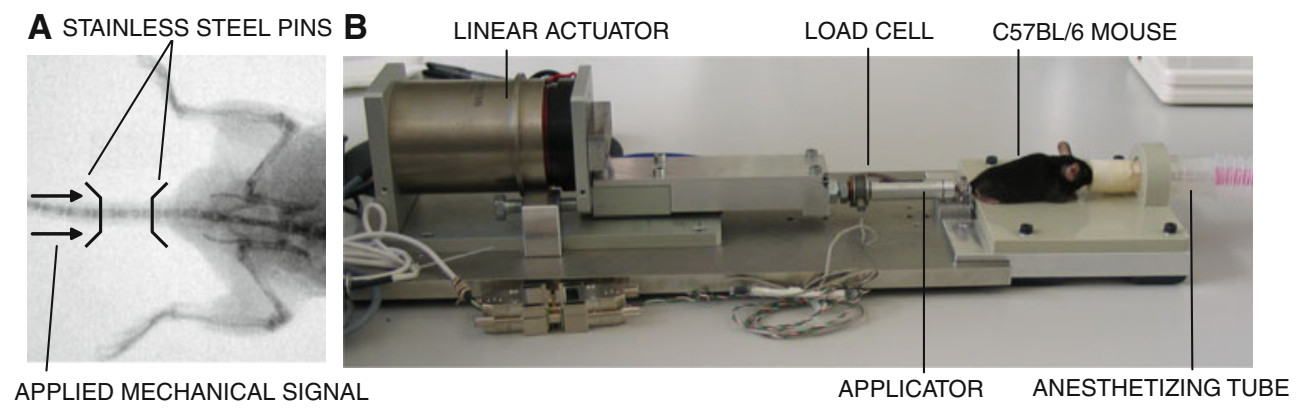

Fig. 1 a Fluoroscopic image of a mouse, graphically edited to show the location and form of the stainless steel pins once they have been surgically inserted. The mechanical signal is applied to the distal pin whilst the proximal pin is clamped. b One loading axis of the Caudal Vertebra Axial Compression Device (CVAD)
Heidelberg, Germany) surgically inserted into their C4 and C6 vertebrae and given a further 3 weeks to recover before loading commenced at 15 weeks of age. For loading, the mice were divided into 8 loading groups $(n=10): \mathrm{B} 6_{0 \mathrm{~N}} / 2 \mathrm{~N} / 4 \mathrm{~N} / 8 \mathrm{~N}$

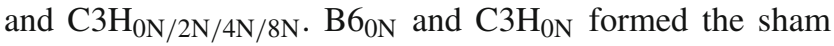
groups. Sham mice, similarly pinned, were mounted into the device and given the same amount of anaesthesia for a period of time equivalent to that of the loaded groups, however, no force was applied to their C5 caudal vertebrae. The remaining groups were submitted to an intensive loading regime whereby sinusoidally varying forces $(10 \mathrm{~Hz})$ were applied to all C5's with amplitudes corresponding to the group subscript. In each loading session, the dynamic mechanical signals were applied for 3,000 cycles and repeated 3 times a week for a duration of 4 weeks (At the end of the experiment the mice were 19 weeks of age). These parameters are of the same order as those used in other loading studies where significant anabolic responses were achieved and which showed a frequency of $10 \mathrm{~Hz}$ to be optimal when loading mouse bone (Rubin et al. 2001; Garman et al. 2007; Ozcivici et al. 2007). Furthermore, prior to the loading experiment in vitro tests performed on pinned tail segments using the protocol defined here did not result in structural failure of the loading configuration.

To vitally label newly formed bone $300 \mu \mathrm{l}$ of the fluorochrome calcein (Sigma, $15 \mathrm{mg} / \mathrm{Kg}$ ) was administered intraperitonally 4 days and 1 day prior to killing. The advantage of employing a short 3-day labelling interval is that it provides a more accurate assessment of the mineralizing perimeter as it minimizes the involvement of osteoclasts and therefore the removal of the calcein labels. This histology protocol has already been successfully applied in other studies measuring bone formation activities (Bajayo et al. 2005; Ofek et al. 2006; Noh et al. 2009). At 19 weeks of age, following 4 weeks of loading all mice were killed using $\mathrm{CO}_{2}$ inhalation. Upon killing of the mice, the C5's were harvested and immediately fixed in formalin for $48 \mathrm{~h}$, after which they were transferred to saline containing $70 \%$ ethanol. Throughout pin insertion and loading the mice were anaesthetized using an oxygen-isoflurane mixture (Provet Medical AG, Lyssach, Switzerland). All animal procedures were approved by the local animal ethics committee (Kantonales Veterinäramt Zürich, Zürich, Switzerland)

\subsection{Quantification of bone adaptation}

Bone morphometry was assessed using micro-computed tomography (micro-CT) with 5 times frame averaging $(6 \mu \mathrm{m}$ voxel size, $50 \mathrm{kVp}, 160 \mu \mathrm{A}$, Scanco Medical AG, Brüttisellen, Switzerland) to obtain 3D, digital images of all C5 vertebrae. Using an algorithm developed earlier (Kohler et al. 2007), cortical and trabecular compartments were isolated (Fig. 2a). To quantify the effect of mechanical loading full morphometric analysis was performed for trabecular and cortical volumes of interest. The trabecular volume of interest was defined by the upper (distal) and lower (proximal) $40 \%$ of the trabecular compartment, whilst the cortical volume of interest was defined as the cortical structure predominantly aligned with the axis of loading (Fig. 2a). Using a direct 3D approach (Hilderbrand 1999), the morphometric indices determined were: Trabecular bone volume density (Tb.BV/TV), trabecular bone volume (Tb.BV), trabecular tissue volume (Tb.TV), trabecular thickness (Tb.Th), trabecular number (Tb.N), cortical bone volume density (Ct.BV/TV), cortical bone volume (Ct.BV), cortical tissue volume (Ct.TV), cortical thickness (Ct.Th) and marrow volume (Ct.MV), where marrow volume describes the volume enclosed by the cortical shell. It should be noted here that cortical bone volume density ( $\mathrm{Ct} . \mathrm{BV} / \mathrm{TV})$ is not a measure of cortical porosity but a measure of the amount of bone within the volume occupied by the cortical shell and the volume that it encloses. To investigate how load-induced bone formation varied throughout the loaded vertebrae trabecular and cortical compartments were divided into overlapping sub-regions (Fig. 2b, c). Bone volume (BV) was determined for all regions. For trabecular bone, the height 


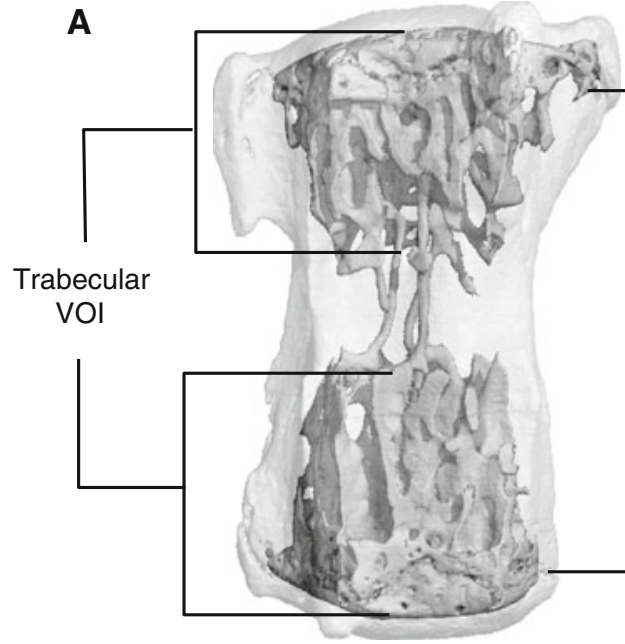

Fig. 2 a Digital image of a whole B6 vertebra (C5) showing cortical and trabecular components. b B6 trabecular bone subdivided into 19 overlapping regions $\left(1_{\mathrm{Tb}}-19_{\mathrm{Tb}}\right)$. c B6 cortical shell subdivided into

of each sub-region was equivalent to $10 \%$ of the height of the total trabecular compartment. Cortical regions were then defined by sub-dividing the cortical shell to match trabecular regions 3-17.

Histomorphometric indices for trabecular and cortical regions were quantified using histological techniques for trabecular, periosteal-cortical (Pe) and endosteal-cortical (En) surfaces. Both single labelled and doubled labelled surfaces were measured. The mineralizing surface of bone per unit of bone surface (Tb.MS/BS, Ct.MS/BS $\mathrm{Pe}_{\mathrm{En}}$ : a surrogate of osteoblast number) was calculated as the double labelled surface plus half of the single labelled surface all divided by the total bone surface. Mineral appositional rate (Tb.MAR, Ct.MARPe/En: a surrogate of osteoblast activity) was obtained by dividing the average distance between the double labels on the bone surfaces by the number of days between injections. In addition, bone formation rates

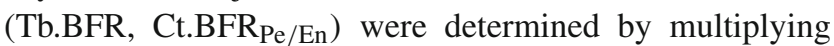
MS/BS and MAR. All indices were determined from 3 single histological slices taken from the mid-sagittal plane of each double labelled vertebra, each 5 micron thick. Tartrate Resistant Acid Phosphatase (TRAP) staining was also performed on adjacent sections to measure the number of osteoclasts present per unit of trabecular perimeter (Tb.N.Oc/Pm). The nomenclature assigned to the histomorphometric indices

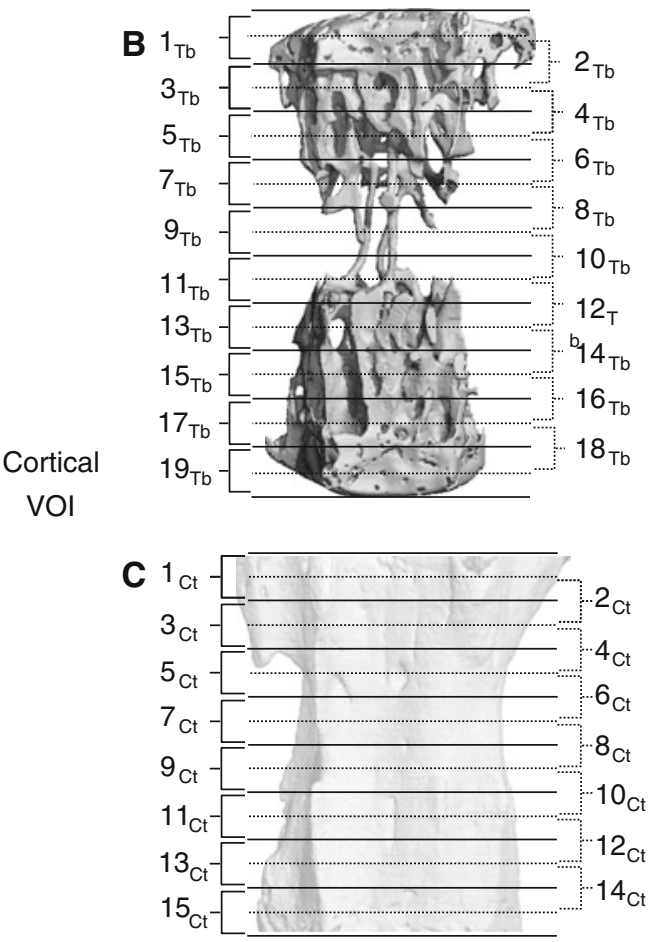

15 overlapping regions $\left(1_{\mathrm{Ct}}-15_{\mathrm{Ct}}\right)$, which match those defining the trabecular regions $3_{\mathrm{Tb}}-17_{\mathrm{Tb}}$

comply with the standards outlined by ASBMR Histomorphometry Nomenclature Committee (Parfitt 1988).

\subsection{Statistical analysis}

To investigate the effect of mechanical loading on each mouse strain linear regression was used to characterize the relationship between applied load and the associated increase in bone volume for both trabecular and cortical volumes of interest. Furthermore, ANOVA was used to compare CT-measured morphometric parameters and dynamic histomorphometric parameters for each loading group. Where significance was detected Fisher's Least Significant Difference was used to determine the significance of the differences between individual loading groups. To compare the relative mechano-sensitivity of the two mouse strains, twoway ANOVA was used to compare their dose responses as measured by both micro-CT and histomorphometry. Owing to the difference in bone size between the two strains, the micro-CT measured dose responses were first normalized using their respective $0 \mathrm{~N}$ loading groups. Again where significance was detected Fisher's Least Significant Difference was used to determine the significance of inter-strain differences for common loads. For all statistical analyses, the 

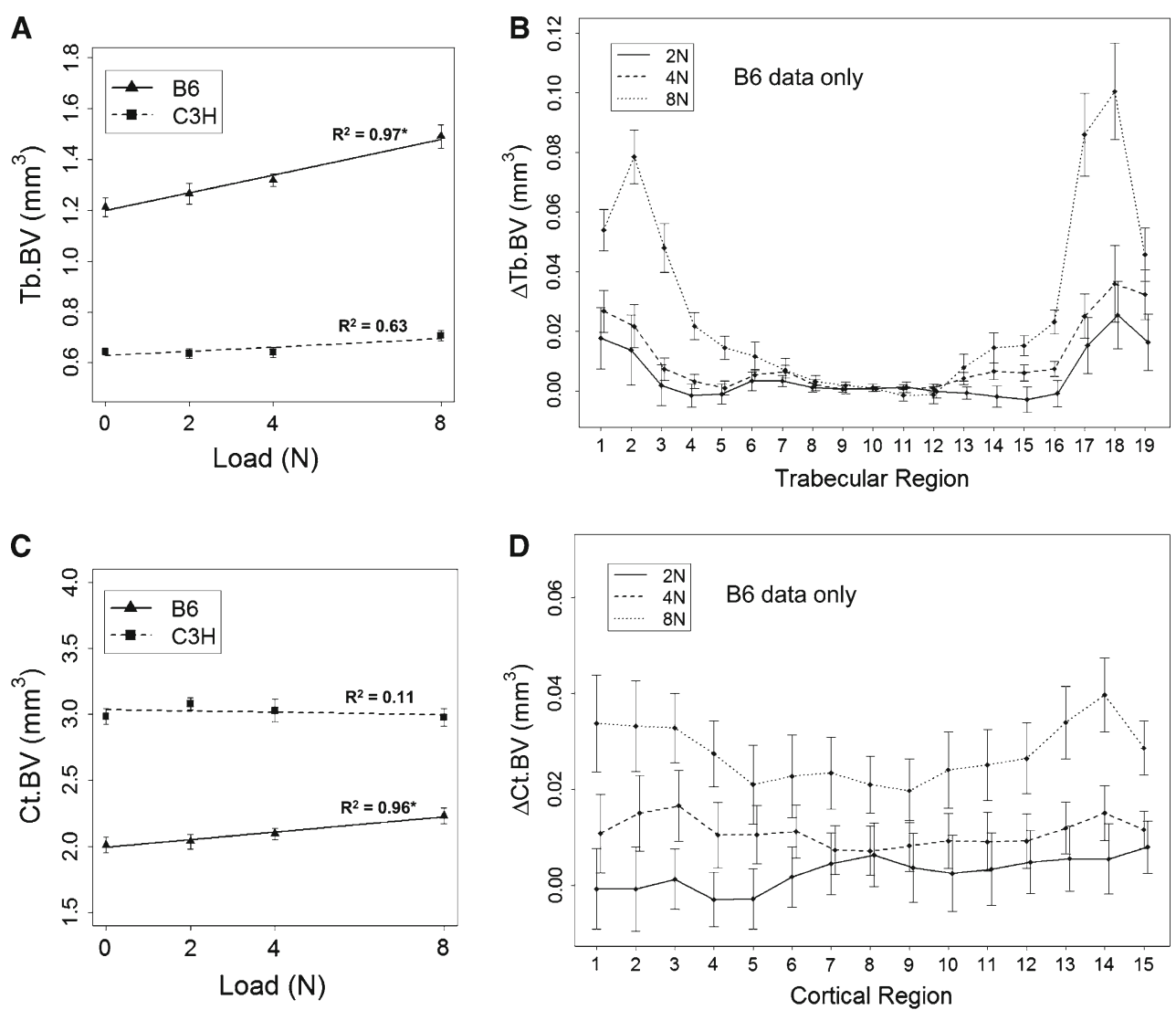

Fig. 3 a The average values of absolute Tb.BV for each loading group plotted against load. b Mean regional absolute increases in trabecular bone mass $(\triangle \mathrm{Tb} . \mathrm{BV})$ in $\mathrm{B} 6$ mice for applied loads of $2 \mathrm{~N}, 4 \mathrm{~N}$ and $8 \mathrm{~N}$. c The average values of absolute Ct.BV for each loading group plotted

against load. $\mathbf{d}$ Mean regional absolute increases in cortical bone mass $(\triangle \mathrm{Ct} . \mathrm{BV})$ in B6 mice for applied loads of $2 \mathrm{~N}, 4 \mathrm{~N}$ and $8 \mathrm{~N}$. * Trend reported by linear regression analysis is significant $(P<0.05)$

statistical package SPSS (Version 17, http://www.spss.com) was used. Results were considered statistically significant for adjusted P-values lower than 0.05 .

\section{Results}

\subsection{Trabecular bone adaptation: B6 mice}

Linear regression analysis (Fig. 3a) showed that Tb.BV increased significantly with load for the B6 strain $\left(R^{2}=\right.$ 0.97). This significant dose response was confirmed by ANOVA. When comparing the micro-CT data sets for all B6 loading groups highly significant differences in Tb.BV/TV, Tb.BV and Tb.Th $(P<0.05)$ were detected. Post hoc analysis revealed that significant increases in Tb.BV/TV and occur for loads of $4 \mathrm{~N}$ and $8 \mathrm{~N}$ when compared to the sham (Table 1). Furthermore, the increases reported for a load of $8 \mathrm{~N}$ were significantly greater than the increases reported for $4 \mathrm{~N}$. The increase in bone mass observed for a load of $8 \mathrm{~N}$ corresponded to a significant increase of $24.4 \%$ in Tb.Th. No significant increases in Tb.N were observed. When analyzing the var- iation of trabecular bone volume throughout the different sub-regions for the all doses (Fig. 3b) it can be seen that most of the anabolic activity occurs at distal and proximal sites whilst there is little or no response in trabeculae at central regions.

A response to mechanical loading in B6 mice was confirmed by histology, the results of which are shown in Table 2 . ANOVA detected significant differences for mineralizing perimeter (Tb.MS/BS), mineral apposition rate (Tb.MAR) and bone formation rate (Tb.BFR). Both Tb.MS/BS and Tb.BFR were found to increase significantly for all loading groups when compared to the sham. However, these increases do not occur in a dose-dependent manner, i.e. the effects are statistically similar for all loads and do not increase with increasing load. Analyses of the TRAP measurements show osteoclast number to decrease significantly for all loads, again in a non-linear fashion.

\subsection{Trabecular bone adaptation: $\mathrm{C} 3 \mathrm{H}$ mice}

When analyzing micro-CT data for $\mathrm{C} 3 \mathrm{H}$ loading groups linear regression showed $\mathrm{BV}$ to increase non-significantly with 


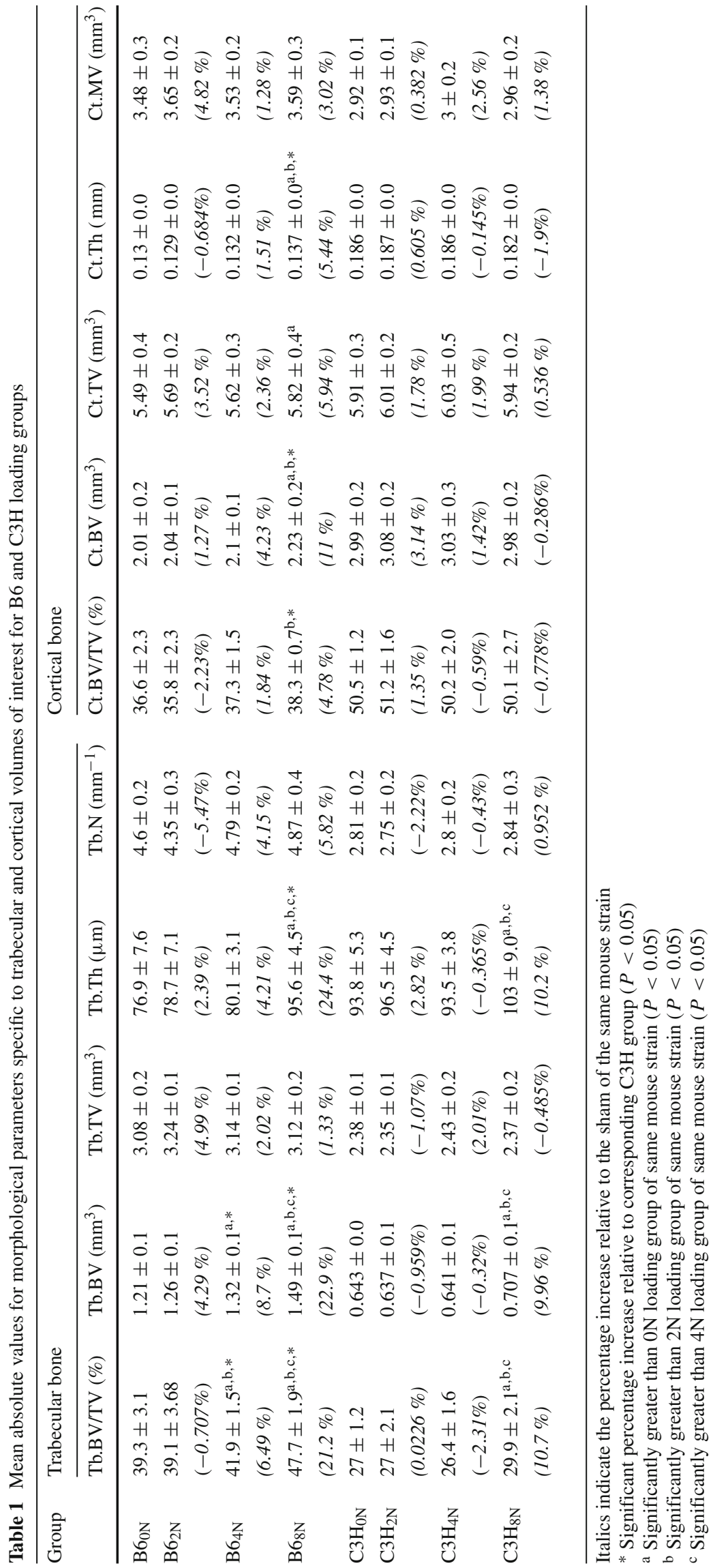




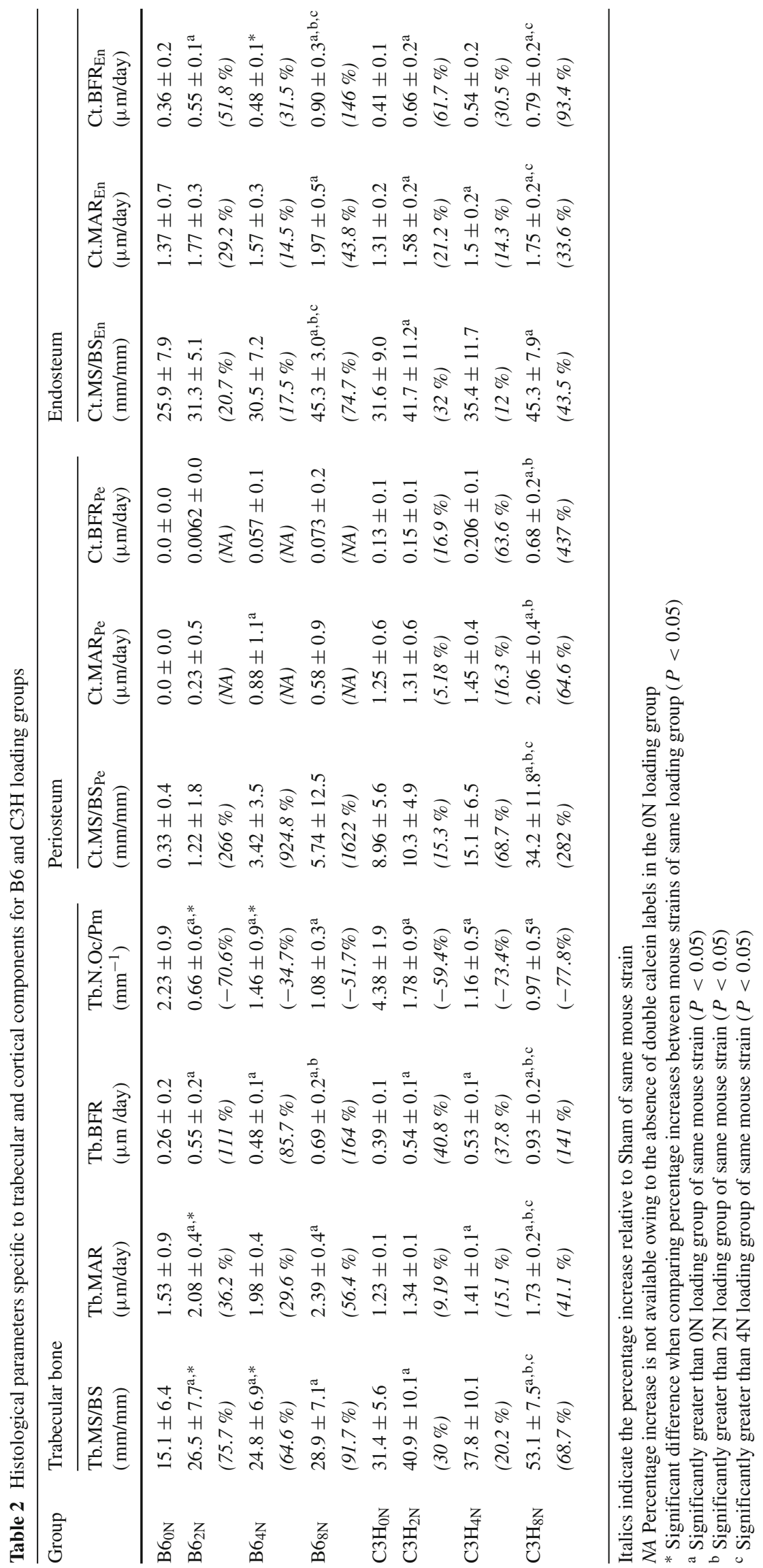


increasing load ( $R^{2}=0.63$, Fig. 3a). ANOVA detected significant effects for a load of $8 \mathrm{~N}$ only. Tb.BV/TV, Tb.BV and $\mathrm{Tb}$.Th were all seen to increase by approximately $10 \%$ when compared to the sham (Table 1). Variation in loadinduced trabecular bone mass with sub-region was shown to be similar to that for B6 mice although much less pronounced (data not shown). Despite the lack of load-induced bone mass as reported by micro-CT, analysis of the histomorphometric indices shows significant increases in BFR for $2 \mathrm{~N}, 4 \mathrm{~N}$ and $8 \mathrm{~N}$ loading groups, albeit in a non-linear fashion. Significant reductions in osteoclast number were also observed for all $\mathrm{C} 3 \mathrm{H}$ loading groups (Table 2).

\subsection{Trabecular bone adaptation: inter-strain comparison}

Inter-strain comparison of micro-CT-based morphometry shows that percentage increases in $\mathrm{Tb} . \mathrm{BV} / \mathrm{TV}$ and $\mathrm{Tb} . \mathrm{BV}$ are significantly higher for the B6 strain when loaded at $4 \mathrm{~N}$ and $8 \mathrm{~N}$ (Table 1). Percentage increases in Tb.Th are significantly higher for the $\mathrm{B} 6$ strain for a load of $8 \mathrm{~N}$ only. The percentage increases reported for histological parameters show cellular activity to be higher in the B6 strain (significant for loads of $2 \mathrm{~N}$ and $4 \mathrm{~N}$ only, Table 2). When considering the osteoclast number, decreases are shown to be significantly greater in the $\mathrm{C} 3 \mathrm{H}$ strain for a load of $4 \mathrm{~N}$ and significantly greater in the B6 strain for a load of $2 \mathrm{~N}$ (Table 2).

\subsection{Cortical bone: B6 mice}

Linear regression analysis showed Ct.BV to increase significantly with increasing load for B6 mice $\left(R^{2}=0.96\right.$, Fig. 3c). When comparing all B6 loading groups ANOVA detected significant differences for Ct.BV/TV, Ct.BV, Ct.TV and Ct.Th. Post hoc analysis reveals that for Ct.BV, Ct.TV and Ct.Th there were significant increases for a load of $8 \mathrm{~N}$ only when compared to the sham (Table 1). Regional analysis of the cortical shell demonstrates a relatively homogeneous response to mechanical loading across all regions when compared to trabecular analysis (Fig. 3d)

Histology shows mineralizing surfaces to increase with increasing load at periosteal cortical sites in B6 mice (Table 2); however, these increases were shown not to be significant. Owing to the absence of double calcein labels at periosteal-cortical sites in the $\mathrm{B}_{0 \mathrm{~N}}$ group percentage increases in Ct.MAR $\mathrm{Pe}_{\mathrm{Pe}}$ and Ct.BFR $\mathrm{Pe}_{\mathrm{P}}$ could not be calculated. At endosteal cortical sites, histomorphometric indices were shown not to increase progressively with increasing load (Table 2). A significant increase was detected in mineralizing surface and mineral apposition rate for a load of $8 \mathrm{~N}$. Increases in bone formation rate were significant for loads of $2 \mathrm{~N}$ and $8 \mathrm{~N}$ when compared to the B6 sham.

\subsection{Cortical bone: $\mathrm{C} 3 \mathrm{H}$ mice}

Linear regression analysis for $\mathrm{C} 3 \mathrm{H}$ mice showed Ct.BV not to increase with increased loading (Fig. 3c). Furthermore, ANOVA found no significant differences for any parameter, as measured by micro-CT (Table 1). Regional analysis supported the lack of activity, showing little or no variation in cortical bone formation for any region for any loading group (regional data not shown). Analysis of the histomorphometric indices shows MS/BS, MAR and BFR to increase progressively at periosteal-cortical sites with increasing load (Table 2). However, significance is detected only for a load of $8 \mathrm{~N}$. At endosteal-cortical sites, similar progressive increases

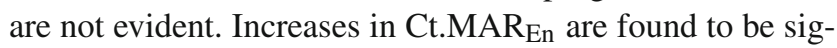
nificant for all loads, whilst increases in Ct.MS/BS $\mathrm{En}$ and $\mathrm{Ct} \mathrm{BFR}_{\mathrm{En}}$ are significant for loads of $2 \mathrm{~N}$ and $8 \mathrm{~N}$.

\subsection{Cortical bone adaptation: inter-strain comparison}

Inter-strain comparison of micro-CT data for cortical bone shows that increases in Ct.BV/TV, Ct.BV and Ct.Th are significantly greater in B6 mice for a load of $8 \mathrm{~N}$ (Table 1). With respect to histomorphometry, all percentage increases are greater in $\mathrm{B} 6$ mice, however significance is only detected for Ct.BFR $\mathrm{En}$ for a load of $4 \mathrm{~N}$ (Table 2).

\section{Discussion}

In this study, we have shown mechanical loading to stimulate bone formation in both trabecular and cortical compartments of B6 female caudal vertebrae. The significant, linearly increasing trend of trabecular bone volume with dose $\left(R^{2}=0.97\right)$ combined with the statistically significant differences in bone mass detected for $0 \mathrm{~N}, 4 \mathrm{~N}$ and $8 \mathrm{~N}$ loading groups demonstrates that load-induced trabecular bone adaptation is dose dependent. Trabecular dynamic histomorphometry was shown not to reflect the dose response detected by micro-CT, nevertheless significantly elevated values of mineralizing surface and bone formation rates for all loads shows that a response mechanism has been activated. The incongruence between micro-CT and histology can be explained by: (1) the difference in time spans covered by the two methods of measurement and (2) the cross-sectional nature of the study. First, histology is a marker of bone formation activity during the last three days of loading, and micro-CT provides a measure of the complete history of all dynamic bone forming activity, hence the rate of deposition of new bone may in some way vary throughout the 4 week loading period. Secondly, whilst trabecular mass is similar between mice, its spatial organization is highly variable. This variability will also be reflected in the loading of individual trabeculae. It is therefore feasible that 3 longitudinal sections from the mid-sagittal 
plane have not captured, on average, the principle regions of bone modelling. Load-induced cortical bone adaptation was also shown to be dose dependent in B6 cortical bone. Whilst statistical comparisons of micro-CT data for the individual loading groups revealed significant increases in cortical bone mass for a load of $8 \mathrm{~N}$ only, regression analysis showed bone volume to increase linearly with dose $\left(R^{2}=0.96\right)$.

To our knowledge, this is the first time a dose response has been demonstrated in loaded, murine trabecular bone. Two other studies using a murine tibia loading model (De Souza et al. 2005; Fritton et al. 2005) have shown cancellous bone to respond to mechanical stimuli; however, the reported effects were not as pronounced. De Souza et al. (2005) was unable to show a significant anabolic effect between loading groups when loading 8-week-old B6 mice, however, a 37\% increase in trabecular BV/TV was achieved, only, when combining the data from all loading groups. Furthermore, in the same study, application of the loading regimes to 12 and 20-week-old mice resulted in a decrease in trabecular bone mass. In another study, Fritton et al. (2005) reported similar $15 \%$ increases in tibial trabecular BV/TV for two groups of male B6 mice (10 weeks of age). These mice were loaded for 2 and 6 weeks using the same mechanical signal. As a reminder, the increases seen in trabecular BV/TV in the loading study presented in this paper were 6.5 and $21.2 \%$ for load amplitudes of $4 \mathrm{~N}$ and $8 \mathrm{~N}$ respectively.

Models requiring surgical intervention have often been criticized for the potential for RAP (regional acceleratory phenomenon) whereby injury to the bone accelerates bone remodeling in neighbouring bones. To address this issue a pilot study was performed prior to this study to characterize the effect of surgical intervention on the morphology of the C5 caudal vertebrae throughout the course of the proposed loading study (data not shown). Using micro-computed tomography, no significant differences in C5 morphology were found to exist between surgical and non-surgical groups killed just before and just after the intended period of loading. Furthermore, there were no significant differences between groups of sham mice killed prior to loading and after the period of loading, demonstrating that the mice used had reached skeletal maturity at vertebral sites. This data is consistent with a study performed by Boyd et al. (Buie et al. 2008), who showed the architectural development in the vertebrae of B6 mice to stabilize after 13 weeks of age.

The comparative lack of load-stimulated trabecular bone mass observed in the $\mathrm{C} 3 \mathrm{H}$ strain, as shown by micro-CT, shows that the response of trabecular and cortical bone to mechanical loading is genotype dependent. Percentage increases in trabecular bone mass are significantly greater for the $\mathrm{B} 6$ strain than those reported for the $\mathrm{C} 3 \mathrm{H}$ strain for loads of $4 \mathrm{~N}$ and $8 \mathrm{~N}$ whilst a significantly greater amount of cortical bone mass was stimulated in B6 mice for a load $8 \mathrm{~N}$ only. In terms of the histological data presented, responses are similar between $\mathrm{B} 6$ and $\mathrm{C} 3 \mathrm{H}$ mice. These data contradict those reported by micro-CT. However, considering that histology is a marker of bone forming activity during the last three days of loading and that micro-CT provides the complete history of all dynamic bone forming activity this discrepancy alludes to either (1) a delayed response in the $\mathrm{C} 3 \mathrm{H}$ strain or (2) non-linear transient patterns of bone forming activity throughout the 4 weeks of loading. The dependence on genotype can be directly attributed to one of two genetically determined factors: (1) Structural differences. It is feasible that for equivalent axial loads the microstrains induced in $\mathrm{C} 3 \mathrm{H}$ vertebrae are lower than those induced in B6 vertebrae, hence there is a reduced mechanical stimulus or (2) Mechano-sensitivity. The response of B6 mice per unit of mechanical strain is higher than that for $\mathrm{C} 3 \mathrm{H}$ mice. This issue is difficult to resolve from the data presented in this paper. From Table 1, it could be deduced that the mechanical strains are higher in B6 mice owing to a small cortical volume and thinner trabeculae, thus structural differences would explain the different responses. However, the trabeculae in B6 mice are greater in number furthermore we do not know how the load is distributed between cortical and trabecular components. To answer these questions additional experimental-based studies, which quantify mechanical strains in both cortical and trabecular compartments, are required i.e. strain gages and finite element modelling. Nevertheless, existing literature attributes genotype-dependent mechano-sensitivities to the different load-induced responses. Kesavan et al. (2005) demonstrated that B6 mice were more receptive to mechanical loading compared to $\mathrm{C} 3 \mathrm{H}$ mice when loading their tibias using a 4 point bending modality even though higher mechanical strains were induced in $\mathrm{C} 3 \mathrm{H}$ mice. $\mathrm{B} 6$ cortical thickness was shown to increase by $28 \%$ compared to $<10 \%$ in $\mathrm{C} 3 \mathrm{H}$ mice, when loaded for 6 days/week over a period of 2 weeks (Peak force: 9N, frequency: $2 \mathrm{~Hz}$ ). A similar effect was shown by Robling and Turner (2002) when loading B6 and C3H ulna. Cortical bone in $\mathrm{C} 3 \mathrm{H}$ mice was shown to have a higher osteogenic threshold $(2,392 \mu \varepsilon)$ compared to that for B6 $(1,769 \mu \varepsilon)$, furthermore once the osteogenic threshold was exceeded cortical bone formation per unit increase in mechanical strain was significantly less for $\mathrm{C} 3 \mathrm{H}$ mice. Other studies supporting the view that B6 mice are more mechano-sensitive than their C3H counterpart include: (Akhter et al. 1998) and (Kuruvilla et al. 2008)

The model presented here has several limitations. First, even though the mice have reached skeletal maturity they are still relatively young, thus the data presented here may not be representative of the response in aged mice. Secondly, a potential injury response was not investigated. Whilst a pilot study found surgical intervention to have no effect on the morphology of C5 vertebrae, it is possible that fatigue induced damage in $\mathrm{C} 4$ and $\mathrm{C} 6$ caudal 
vertebrae could. There were no external signs of fatigue related damage, nevertheless in future experiments both C4 and C6 caudal vertebrae should be analysed for damage, this would involve scanning the pinned vertebrae using micro-CT and searching for micro damage. Thirdly, owing to the cross-sectional nature of the study and the protocol chosen for histomorphometry, the histological data lacks specificity. Whilst trabecular content is similar between mice, its spatial organization is highly variable. This variability will also be reflected in the loading of individual trabeculae. It is therefore feasible that 3 longitudinal sections from the mid-sagittal plane have not captured the principle regions of bone modelling. To better identify the exact spatial and temporal patterns of bone modelling future studies should make use of in vivo imaging technologies (Boyd et al. 2006; Lambers et al. 2009), a technology which is particularly suited to the imaging of caudal vertebrae given their accessible anatomical location.

Although invasive, compared to the tibia loading model, the proposed model has the advantage that much more trabecular bone can be analysed. This will prove useful in future studies, which investigate load-regulated genes as the sensitivity of current gene detection technologies is limited by the amount of input material. Furthermore, owing to the size and shape of the C5 caudal vertebrae the proposed loading configuration will result in a much simplified micromechanical environment i.e. the presence of bending stresses and shear stresses will be minimized, which is not the case with long bones. This simplified modality of compression will enable us to more easily characterize the spatial relationship between load- induced bone adaptation the associated micromechanical environment.

\section{Conclusion}

In performing this study, we have demonstrated that we can surgically insert stainless steel pins into the $\mathrm{C} 4$ and $\mathrm{C} 6$ vertebrae of both $\mathrm{B} 6$ and $\mathrm{C} 3 \mathrm{H}$ mice, we have also shown that we can accurately apply dynamic mechanical loads to their C5 vertebrae for a sustained period of time, whilst inducing a significant dose response in the cortical and trabecular bone of B6 mice. We have also shown that load-induced bone adaptation is genotype dependent. The availability of this approach provides an alternative to the tibia loading model thus affording opportunities to explore site specific mechano-sensitivity in both cortical and trabecular bone. Moreover, the dosedependent nature of load-induced bone formation will allow us to gain further insight into the underlying mechanical parameters and molecular process governing bone formation when combined with in vivo imaging technologies, finite element analysis and suitable methods of biochemical analysis.

\section{References}

Akhter MP, Cullen DM et al (1998) Bone response to in vivo mechanical loading in two breeds of mice. Calcif Tissue Int 63(5):442-449

Albright J (1987) The scientific basis of orthopaedics, 2nd edn. Appleton-Century Crofts, New York

Bajayo A, Goshen I et al (2005) Central IL-1 receptor signaling regulates bone growth and mass. Proc Natl Acad Sci USA 102(36):12956-12961

Beamer WG, Shultz KL et al (2001) Quantitative trait loci for femoral and lumbar vertebral bone mineral density in C57BL/6J and $\mathrm{C} 3 \mathrm{H} / \mathrm{HeJ}$ inbred strains of mice. J Bone Miner Res 16(7):11951206

Biewener AA, Fazzalari NL et al (1996) Adaptive changes in trabecular architecture in relation to functional strain patterns and disuse. Bone 19(1):1-8

Boyd SK, Davison P et al (2006) Monitoring individual morphological changes over time in ovariectomized rats by in vivo micro-computed tomography. Bone 39(4):854-862

Buie HR, Moore CP et al (2008) Postpubertal architectural developmental patterns differ between the L3 vertebra and proximal tibia in three inbred strains of mice. J Bone Miner Res 23(12):20482059

Chambers TJ, Evans M et al (1993) Induction of bone formation in rat tail vertebrae by mechanical loading. Bone Miner 20(2):167-178

De Souza RL, Matsuura M et al (2005) Non-invasive axial loading of mouse tibiae increases cortical bone formation and modifies trabecular organization: a new model to study cortical and cancellous compartments in a single loaded element. Bone 37(6):810-818

Duncan RL, Turner CH (1995) Mechanotransduction and the functional response of bone to mechanical strain. Calcif Tissue Int 57(5):344358

Evans WJ (1998) Exercise and nutritional needs of elderly people: effects on muscle and bone. Gerodontology 15(1):15-24

Fritton JC, Myers ER et al (2005) Loading induces site-specific increases in mineral content assessed by microcomputed tomography of the mouse tibia. Bone 36(6):1030-1038

Frost HM (1987) The mechanostat: a proposed pathogenic mechanism of osteoporoses and the bone mass effects of mechanical and nonmechanical agents. Bone Miner 2(2):73-85

Garman R, Gaudette G et al (2007) Low-level accelerations applied in the absence of weight bearing can enhance trabecular bone formation. J Orthop Res 25(6):732-740

Guo XE, Eichler MJ et al (2002) Quantification of a rat tail vertebra model for trabecular bone adaptation studies. J Biomech 35(3): 363-368

Hilderbrand T (1999) Direct three-demensional morphometric analysis of human cancellous bone: microstructural data from spine, femur, iliac crest and calcaneus. J Bone Miner Res 14:1167-1174

Kesavan C, Mohan S et al (2005) Mechanical loading-induced gene expression and BMD changes are different in two inbred mouse strains. J Appl Physiol 99(5):1951-1957

Kesavan C, Mohan S et al (2006) Identification of genetic loci that regulate bone adaptive response to mechanical loading in C57BL/6J and $\mathrm{C} 3 \mathrm{H} / \mathrm{HeJ}$ mice intercross. Bone 39((3):634-643

Kodama Y, Umemura Y et al (2000) Exercise and mechanical loading increase periosteal bone formation and whole bone strength in $\mathrm{C} 57 \mathrm{BL} / 6 \mathrm{~J}$ mice but not in $\mathrm{C} 3 \mathrm{H} / \mathrm{Hej}$ mice. Calcif Tissue Int 66(4):298-306

Kohler T, Stauber M et al (2007) Automated compartmental analysis for high-throughput skeletal phenotyping in femora of genetic mouse models. Bone 41(4):659-667

Koller DL, Schriefer J et al (2003) Genetic effects for femoral biomechanics, structure, and density in C57BL/6J and C3H/HeJ inbred mouse strains. J Bone Miner Res 18(10):1758-1765 
Kuruvilla SJ, Fox SD et al (2008) Site specific bone adaptation response to mechanical loading. J Musculoskelet Neuronal Interact 8(1):71-78

Lambers FM, Kuhn G et al (2009) In vivo micro computed tomography allows monitoring of load induced microstructural bone adaptation. Bone 44(S2) (in press)

Lau KH, Kapur S et al (2006) Up-regulation of the Wnt, estrogen receptor, insulin-like growth factor-I, and bone morphogenetic protein pathways in $\mathrm{C} 57 \mathrm{BL} / 6 \mathrm{~J}$ osteoblasts as opposed to $\mathrm{C} 3 \mathrm{H} / \mathrm{HeJ}$ osteoblasts in part contributes to the differential anabolic response to fluid shear. J Biol Chem 281(14):9576-9588

Marcus R (1995) Relationship of age-related decreases in muscle mass and strength to skeletal status. J Gerontol A Biol Sci Med Sci 50 Spec No: $86-87$

Noh T, Gabet Y et al (2009) Lef1 haploinsufficient mice display a low turnover and low bone mass phenotype in a gender- and age-specific manner. PLoS One 4(5):e5438

Ofek O, Karsak M et al (2006) Peripheral cannabinoid receptor, CB2, regulates bone mass. Proc Natl Acad Sci USA 103(3):696-701

Ozcivici E, Garman R et al (2007) High-frequency oscillatory motions enhance the simulated mechanical properties of non-weight bearing trabecular bone. J Biomech 40(15):3404-3411
Parfitt AM (1988) Bone histomorphometry: proposed system for standardization of nomenclature, symbols, and units. Calcif Tissue Int 42(5):284-286

Robling AG, Turner CH (2002) Mechanotransduction in bone: genetic effects on mechanosensitivity in mice. Bone 31(5):562-569

Rubin CT, Lanyon LE (1985) Regulation of bone mass by mechanical strain magnitude. Calcif Tissue Int 37(4):411-417

Rubin CT, Sommerfeldt DW et al (2001) Inhibition of osteopenia by low magnitude, high-frequency mechanical stimuli. Drug Discov Today 6(16):848-858

Turner $\mathrm{CH}$, Hsieh YF et al (2000) Genetic regulation of cortical and trabecular bone strength and microstructure in inbred strains of mice. J Bone Miner Res 15(6):1126-1131

Webster DJ, Morley PL et al (2008) A novel in vivo mouse model for mechanically stimulated bone adaptation-a combined experimental and computational validation study. Comput Methods Biomech Biomed Engin 11(5):435-441

Xing W, Baylink D et al (2005) Global gene expression analysis in the bones reveals involvement of several novel genes and pathways in mediating an anabolic response of mechanical loading in mice. J Cell Biochem 96(5):1049-1060 